
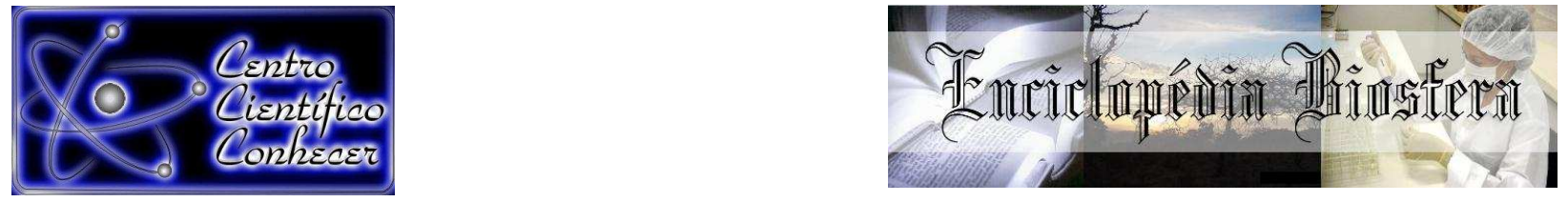

\title{
AVALIAÇÃO DA QUALIDADE DE VIDA DE PACIENTES RENAIS CRÔNICOS EM HEMODIÁLISE
}

Luciana de Souza Ondei ${ }^{1}$; Francilene Alves Ribeiro ${ }^{2}$; Vitória Regina Moreira de Noronha $^{2}$; Fabrício Barreto Teresa ${ }^{3}$

${ }^{1}$ Profa. Doutora da Universidade Estadual de Goiás, Câmpus Anápolis de Ciências Exatas e Tecnológicas (luondei@yahoo.com.br), Anápolis, GO - Brasil.

${ }^{2}$ Biólogas pela Universidade Estadual de Goiás, Câmpus Porangatu, Porangatu, GO - Brasil.

${ }^{3}$ Prof. Doutor da Universidade Estadual de Goiás, Câmpus Anápolis de Ciências Exatas e Tecnológicas, Anápolis, GO - Brasil.

Recebido em: 03/10/2016 - Aprovado em: 21/11/2016 - Publicado em: 05/12/2016 DOI: 10.18677/EnciBio_2016B_130

No Brasil, a elevada incidência de pacientes renais crônicos é considerada um dos principais problemas de saúde por estar relacionada a um alto custo, morbidade e mortalidade. Na fase mais avançada da doença, torna-se necessária a hemodiálise que visa à melhora da qualidade de vida (QV), mas ao mesmo tempo expõe o paciente a complicações clínicas e psicológicas que afetam diretamente sua QV. Este trabalho objetivou avaliar a QV de pacientes renais crônicos $(\mathrm{N}=30)$, com base em diversos indicadores dispostos em um questionário composto por 20 perguntas objetivas e cinco discursivas. A partir dos resultados obtidos, contatou-se que $67 \%$ dos entrevistados apresentavam comorbidades que não demonstraram influência sobre a QV $(p=0,68)$. As mulheres mostraram-se mais aptas ao trabalho que os homens $(p=0,03)$. Não houve influência da idade sobre a QV $(p>0,05)$. No entanto, as questões discursivas indicaram que os idosos encaram o tratamento de forma mais positiva que os demais grupos. O tempo de exposição ao tratamento mostrouse bastante significativo, sendo que tanto a frequência de sentimentos negativos $(p=0,04)$ como o sentimento de dependência do tratamento $(p=0,02)$ foi maior nos pacientes em hemodiálise há mais de três anos. De forma geral, a QV dos pacientes é considerada boa não havendo influência da maioria dos indicadores analisados.

PALAVRAS-CHAVE: Diálise renal, Insuficiência renal, Nefrologia

\section{QUALITY OF LIFE IN PATIENTS WITH CHRONIC KIDNEY DISEASE UNDERGOING HEMODIALYSIS}

\begin{abstract}
The high incidence of chronic renal patients is considered one of the major health issues in Brazil, being related to a high cost, morbidity and mortality. The hemodialysis is the most utilized treatment in the terminal stage of the disease, aiming to improve the quality of life (QoL), but at the same time exposes the patient
\end{abstract}


to clinical and psychological complications that directly affect their QoL. This study aimed to evaluate the QoL of chronic renal patients $(\mathrm{N}=30)$, based on several indicators arranged in a questionnaire with 20 objective questions and five discursive. Sixty seven percent of respondents had comorbidities that showed no influence on QoL ( $p>0.05)$. Women patients were more likely to work than men $(p=0.03)$. There was no influence of age on QoL ( $p>0.05)$. Elderly persons cope the treatment more positively than the other groups, as shown by discursive questions. The treatment exposure time was associated to both the frequency of negative feelings $(p=0.04)$ and the feeling of treatment dependence $(p=0.02)$ in patients on hemodialysis for more than three years. Overall, patients' QoL is considered good with no influence of most of the indicators analyzed.

KEYWORDS: Renal insufficiency, Renal dialysis, Nephrology

\section{INTRODUÇÃO}

Os rins desempenham funções fundamentais para a manutenção da homeostase corporal, como a eliminação de toxinas do sangue; produção da eritropoietina, hormônio que atua na maturação dos glóbulos vermelhos do sangue e da medula óssea; regulação da pressão sanguínea por meio do sistema reninaangiotensina-aldosterona (RAAS), dentre outras (MEDEIROS \& MEDEIROS, 2013; MARTELLI, 2013). A diminuição da função renal implica no comprometimento de essencialmente todos os outros órgãos e a detecção precoce de alterações renais é extremamente importante para a conduta terapêutica adequada (BASTOS et al., 2010).

A insuficiência renal aguda (IRA) é caracterizada pela perda rápida, porém temporária da função renal sendo necessário, em alguns casos, recorrer à diálise até que os rins voltem a funcionar (LI et al., 2013). Já a insuficiência renal crônica (IRC) caracteriza-se pela perda lenta, progressiva e irreversível da função renal e, na maioria dos casos, o paciente permanece assintomático até que tenha perdido $50 \%$ de sua função renal. A partir desse momento começam a aparecer alguns sintomas que geralmente não incomodam muito, o que prejudica ainda mais o diagnóstico. O paciente pode ser tratado com medicamentos e dieta se sua função renal estiver até $12 \%$. No entanto, se a função renal estiver abaixo destes valores será necessário o uso de outros métodos como a diálise ou o transplante renal (MACHADO \& PINHATI, 2014).

A diabetes e a hipertensão são duas comorbidades altamente associadas com a insuficiência renal e, se não tratadas corretamente, podem contribuir para a falência total do funcionamento dos rins. A detecção precoce dos problemas renais e - acompanhamento apropriado dos doentes podem reduzir os custos com o tratamento e melhorar a qualidade de vida (QV) dos pacientes (VIEIRA JÚNIOR \& SUASSUNA, 2013). A compreensão da doença renal é de extrema importância, tendo em vista sua relação com o aumento na mortalidade e de gastos com o tratamento, bem como a queda da QV dos pacientes que evoluem para a IRC (MARTINS \& CESARINO, 2005).

De acordo com a Sociedade Brasileira de Nefrologia, o transplante renal é indicado para pacientes com IRC em fase terminal que estejam em diálise ou em fase pré-dialítica (SOCIEDADE BRASILEIRA DE NEFROLOGIA, 2016). O transplante renal é considerado um método de tratamento bem aceito do ponto de 
vista médico, social e econômico e, muitas vezes, é a única opção viável em longo prazo (GARCIA-GARCIA et al., 2012). No entanto, por ser um processo demorado, o tratamento dialítico contínuo é uma alternativa para a manutenção da vida do paciente (SOCIEDADE BRASILEIRA DE NEFROLOGIA, 2016).

A diálise é um processo fisiológico pelo qual há a separação de substâncias através de uma membrana permeável. Na hemodiálise o processo de filtração é extracorpóreo no qual o sangue é filtrado por uma membrana semipermeável derivada de celulose que fica imersa em uma solução eletrolítica funcionando como um rim artificial o que garante a sobrevida do paciente. As máquinas de hemodiálise apresentam uma bomba que é responsável pelo movimento extracorpóreo do sangue, um sistema de fornecimento de solução dialítica e monitores de segurança (FERMI, 2003).

O tratamento hemodialítico expõe o paciente renal crônico a diversas complicações clínicas e condições de estresse, provocadas por uma sucessão de situações que comprometem não só o aspecto físico, mas também psicológico, com repercussões pessoais, familiares e sociais (SILVA, 2011; VALLE et al., 2013). Estes fatores desempenham um impacto negativo na $Q V$ relacionada à saúde que, segundo MARTINS \& CESARINO (2005), pode ser caracterizada como a percepção da pessoa sobre sua saúde, por meio de uma avaliação subjetiva de seus sintomas, satisfação e adesão ao tratamento. Os pacientes renais crônicos acabam tornandose desanimados, desesperados e, muitas vezes, por estas razões ou por falta de orientação, acabam abandonando o tratamento ou não dando importância aos cuidados constantes que deveriam ter (LIMA \& GUALDA, 2001). A aceitação, a aderência ao tratamento e o cumprimento das orientações médicas em conjunto com o apoio familiar, a religiosidade, a disponibilidade de informação e a prática de atividades físicas ou de lazer tendem a auxiliar no processo de adaptação do paciente ao tratamento e, consequentemente, elevam sua QV (BARBOSA \& VALADARES, 2009).

A medida da QV é cada vez mais recomendada como parâmetro a ser monitorado regularmente, visando à diminuição no número de mortalidade por meio da compreensão do paciente, tanto durante o seu período de adaptação, como no enfrentamento contínuo e eficaz da doença (GRASSELLI et al., 2012). Sendo assim, a portaria GM/SM № 1168, de 15 de junho de 2004, estabelece a Política Nacional de Atenção ao Portador de Doença Renal, e busca entre outras coisas, desenvolver estratégias que promovam a QV, isto porque a doença renal crônica juntamente com seu tratamento pode atingir o paciente no âmbito físico, psíquico e social (BRASIL, 2004).

A IRC é considerada um dos principais problemas de saúde do mundo e, no Brasil, a elevada incidência de portadores da doença (SESSO et al., 2016) tem assumido uma importância considerável, pois está relacionada a um alto custo, morbidade e mortalidade (DRAIBE, 2014). Nesta perspectiva, o objetivo desse trabalho foi avaliar a QV de pacientes renais crônicos em hemodiálise segundo indicadores sociodemográficos, físicos e aspectos da doença.

\section{MATERIAL E MÉTODOS}

Este estudo trata-se de uma pesquisa transversal quantitativa e qualitativa realizada entre os meses de julho e agosto de 2013, com pacientes renais crônicos 
em tratamento hemodialítico, em uma Clínica de Diálise da cidade de Porangatu/GO. O trabalho foi aprovado pelo Comitê de Ética do Hospital Geral de Goiânia - HGG, com CAAE no 16147113.1.0000.0035 e foi desenvolvido de acordo com os princípios éticos da Resolução 466/2012. A coleta de dados ocorreu após a assinatura do Termo de Consentimento Livre e Esclarecido. O anonimato dos participantes foi preservado por meio do uso de códigos (P1 a P30) para as citações.

Foram incluídos nesta pesquisa pacientes com IRC, que se encontravam no mínimo de um ano em hemodiálise, que não foram submetidos a transplante renal e que tinham mais de 18 anos. Desta forma, participaram 30 pacientes entre 19 e 81 anos.

Com relação à QV avaliou-se aspectos sociodemográficos como sexo, idade e renda familiar; indicador físico como capacidade para o trabalho; e aspectos relacionados à doença como presença de comorbidades, tempo em hemodiálise, frequência de sentimentos negativos, sentimento de dependência, compreensão da necessidade e adaptação ao tratamento, e medidas para minimizar a sobrecarga do tratamento, além da auto avaliação sobre a qualidade de vida e saúde. Estes indicadores foram baseados no protocolo WHOQOL-BREF- World Health Organization Quality of Life Instrument (THE WHOQOL GROUP, 1998). Trata-se de um instrumento simplificado de avaliação da QV desenvolvido pela Organização Mundial de Saúde que considera aspectos de saúde física, psicológica, relacionamento social e aspectos ambientais, já tendo sido validado no Brasil (BERLIM et al., 2005; ROCHA; FLECK, 2009). O questionário foi composto por 25 questões, sendo 20 objetivas com as respostas divididas em escala ordinal (um a cinco) e cinco discursivas complementares explorando aspectos qualitativos relacionados com a rotina e adaptação ao tratamento. As entrevistas foram gravadas e armazenadas em arquivo digital.

\section{Análise estatística}

Os dados obtidos por meio dos questionários foram inseridos em planilha do software Microsoft Excel. Os resultados foram avaliados por meio de testes descritivos e inferenciais. Por se tratarem de dados não paramétricos, foram utilizados os testes de Kruskal-Wallis, quando o objetivo foi verificar a influência de uma variável sobre três ou mais grupos independentes, e o teste de Mann-Whitney, para avaliar a influência de uma variável sobre dois grupos independentes. Os testes foram realizados com o uso do software Statistica7 e o nível de significância adotado foi de $p<0,05$.

\section{RESULTADOS E DISCUSSÃO}

As características sociodemográficas dos pacientes estão apresentadas na Tabela 1. Constatou-se que dos 30 pacientes entrevistados, $53 \%$ eram homens e $47 \%$ eram mulheres. A média de idade foi de $50,1( \pm 16,3)$ anos, variando de 19 a 81 anos. Do total analisado, 33\% eram jovens adultos (19 a 39 anos), 40\% adultos de meia idade (40 a 60 anos) e $27 \%$ eram idosos (mais de 60 anos). Perfil de idade semelhante foi encontrado em outros trabalhos (TELLES et al. 2014; RIBEIRO et al., 2014). O acometimento de pessoas em idade economicamente ativa é um dado significativo, pois geram gastos nas áreas sociais devido às aposentadorias precoces, gastos ambulatoriais e medicamentosos (RIBEIRO et al. 2014). 
TABELA 1: Características sociodemográficas dos pacientes em hemodiálise $(\mathrm{N}=30)$.

\begin{tabular}{llc}
\hline Variável & & Valor percentual \\
\hline \multirow{2}{*}{ Sexo } & Masculino & 53 \\
& Feminino & 47 \\
\hline \multirow{3}{*}{ Idade } & 19 a 39 anos & 33 \\
& 40 a 60 anos & 40 \\
& Mais de 60 anos & 27 \\
\hline \multirow{4}{*}{ Renda familiar } & Até 1 salário mínimo & 47 \\
& Entre 2 e 3 salários mínimos & 37 \\
& Entre 3 e 5 salários mínimos & 13 \\
& Mais de 5 salários mínimos & 3 \\
\hline
\end{tabular}

Não houve influência da idade sobre a QV (Kruskal-Wallis, $p=0,55$ ). Contudo, ao analisar as questões discursivas, verificou-se que os idosos, ao contrário dos demais grupos, apresentavam uma visão positiva em relação à $Q V$ relacionada à saúde e viam a hemodiálise como uma forma de sobrevida: "Hoje eu sou outro né, em relação ao que eu tive hoje eu sou outro, bem melhor [...] Eu aceitei logo viu, foi rápida a minha aceitação" (P1). "A hemodiálise não mata ninguém, só dá vida, não é bom, mas dá vida!' (P2). De fato, a resiliência em relação ao tratamento dos pacientes em idade mais avançada também foi evidenciada em outros estudos. Se por um lado há associação positiva entre a idade e o comprometimento de aspectos físicos como dor e vitalidade, pouco ou nenhum efeito da idade sobre a saúde mental tem sido encontrada (SINGER et al., 1999; SANTOS, 2006).

A renda familiar foi relativamente baixa. Do total analisado, $47 \%$ apresentavam renda de até um salário, $37 \%$ entre dois e três salários, $13 \%$ de três a cinco salários e $3 \%$ dos pacientes tinham renda superior a cinco salários mínimos. Verificou-se que a QV não variou de acordo com a renda (Kruskal Wallis, $p=0,87$ ). Esses resultados podem ser justificados pelo fato do tratamento demandar o mesmo investimento de tempo de todos os pacientes, independente da renda familiar (GRASSELLI et al., 2012). Assim, esses resultados indicam que os efeitos do tratamento afetam igualmente pacientes com rendas bem distintas (SANTOS, 2006).

Observou-se a influência do sexo na capacidade para o trabalho, uma vez que as mulheres em hemodiálise mostraram-se mais aptas do que os homens $(p=0,03)$. Este resultado foi confirmado nos depoimentos em que os homens mostraram-se ressentidos pela impossibilidade de trabalhar, o que dificultava a manutenção das necessidades da família: "O que mudou na minha vida, no caso, não foi nem a minha falta de lazer certo, foi mais pelo meu trabalho [...] Nunca suportei ficar sem trabalhar e aí você passa a sentir, você passa a depender dos filhos da família [...]" (P3). Atribui-se a sobrecarga emocional do tratamento à menor capacidade para o trabalho identificada no sexo masculino, devido ao fato históricocultural no qual o homem é o provedor do sustento familiar. Outros trabalhos também afirmam que a perda das atividades, principalmente do trabalho, são as principais causas de insatisfação dos pacientes (LOPES et al., 2014; LIRA et al., 2015). MADEIRO et al. (2010) observaram, no que diz respeito ao trabalho, que os modos de enfrentamento focados na emoção têm associação positiva com o 
trabalho, pois as pessoas que desenvolvem alguma atividade têm menos depressão e, especialmente, as mulheres são menos ansiosas.

Dentre os entrevistados, $67 \%$ apresentavam alguma comorbidade $(37 \% \mathrm{com}$ hipertensão, $13 \%$ com diabetes, $7 \%$ com nefrite crônica e 10\% com outras comorbidades). Contudo não houve associação com a QV desses pacientes (MannWhitney, $p=0,68$ ). Segundo ALVARES et al., 2013, a análise de comorbidades isoladas mostram fraca relação com a $\mathrm{QV}$, o que pode justificar o resultado encontrado.

Quanto ao tempo em hemodiálise, 37\% dos pacientes estavam em tratamento há um ano, $27 \%$ de dois a três anos e $37 \%$ há mais de três anos. Embora o tempo em hemodiálise não tenha sido associado com a QV (Kruskal-Wallis, $p=0,2$ ), essa variável influenciou a frequência de sentimentos negativos (Kruskal-Wallis, $p=0,04$ ). Pacientes em hemodiálise há mais de três anos apresentavam maior frequência de algum tipo de sentimento negativo, diferente daqueles em tratamento entre um e dois anos. A análise dos depoimentos ratifica estes dados: "Demorou bastante até eu cair na realidade do que estava fazendo... está sendo difícil agora, porque antes eu não achava ruim, tudo estava bom, eu não estava sentindo nada! Não estava sentindo fraqueza, não estava sentindo as ansiedades, não estava ficando nervosa à toa, estressada. Agora tudo me cansa, a hemodiálise me cansa, as pessoas me cansam, eu fico revoltada e tem dias, assim, que eu não quero falar com ninguém, eu não quero ver ninguém [...]" (P4).

A maior frequência de sentimentos negativos com o passar do tempo em que o paciente se encontra sobre as diversas condições estressoras provenientes do tratamento é um fator esperado, visto que o mesmo não apresenta alternativa à cura, sendo a hemodiálise uma forma de garantir sua sobrevida, enquanto aguarda o transplante (BARBOSA \& VALADARES, 2009). Dessa forma, à medida que o tempo passa, acumula-se o estresse, o temor e assim também diminuem as esperanças de uma possível recuperação, geralmente presentes nos primeiros anos.

O sentimento de dependência do tratamento pelo paciente mostrou-se significativamente maior quando relacionado ao tempo em hemodiálise (KruskalWallis, $\mathrm{p}=0,02)$ sendo também observado nas questões discursivas: "Nos primeiros dias a gente ficava assim meio não querendo, mas depois a gente viu que precisava" (P5). "Agora eu sinto falta do tratamento, por exemplo, se eu exagerar um pouquinho na comida, na bebida eu tenho necessidade, então eu sinto falta, eu sinto bem com a hemodiálise" (P6). O aumento do sentimento de dependência do tratamento com o passar do tempo pode ser visto inicialmente como um fator negativo para a QV dos pacientes (GRASSELLI et al., 2012). Contudo, a correlação entre esses fatores indica uma relação positiva. Isto ocorre porque no início do tratamento os pacientes têm uma tendência em não aceitar sua condição de dependência, se recusando a cumprir as orientações médicas e até mesmo as datas estabelecidas para a hemodiálise, acarretando uma QV prejudicada pelas condições da saúde debilitada (MADEIRO et al., 2010). Portanto, verificou-se, que a compreensão da necessidade do tratamento para a manutenção da saúde do paciente renal crônico é fundamental para sua melhor QV (BARBOSA \& VALADARES, 2009; CASTRO \& GROSS, 2013). 
Houve um aumento na QV pela compreensão da necessidade do tratamento (Kruskal-Wallis, $p=0,02$ ). As análises das questões discursivas revelaram que a grande maioria dos entrevistados não conhecia o tratamento que estava iniciando, relatando sentimentos como desespero, depressão e preocupação, principalmente durante o primeiro ano de hemodiálise, em que a adaptação é mais intensa. Outros disseram ter aceitado o tratamento com facilidade, por já terem sido orientados pelos médicos ou por verem a hemodiálise como uma oportunidade de continuar vivendo. Desta forma, observam-se diferentes reações e modos de agir durante o processo de enfrentamento da doença (SILVA et al., 2011).

Portanto, ao serem questionados sobre o período de adaptação e, consequentemente, sobre as mudanças advindas do tratamento, houve relatos tanto positivos quanto negativos, que variaram conforme a visão do paciente. Pode-se observar que os relatos positivos eram provenientes de comparações feitas pelos próprios pacientes, do estágio pré-tratamento, em que eles se encontravam muito debilitados devido à doença e, pós-tratamento, no qual houve uma melhora importante na saúde, conforme relatado: "Minha vida melhorou bastante, 100\%, porque, quando eu saí daqui para fazer esse tratamento [...] tinha dia que me dava tanta fraqueza [...] falta de ar. Depois da hemodiálise melhorou 100\%, hoje graças a Deus, eu me sinto forte o bastante, bem melhor?' (P7).

Alguns pacientes relataram as restrições alimentares, de lazer e até mesmo do trabalho, além das alterações físicas e emocionais: "Muita coisa mudou porque você tem que mudar sua alimentação, sua rotina... mudar toda a sua vida, mudar muita coisa [...] você tem que ter muita regra para a alimentação, bastante coisa que você não pode fazer [...] me deu muito mau humor" (P8). "A pele fica muito escura, não pode trabalhar. O que mais me incomoda é isso, não poder trabalhar" (P9). Desta forma, é possível observar que a QV dos pacientes é afetada negativamente em diferentes dimensões: profissional, vitalidade, função física, função emocional e saúde geral que pode ser justificada pelas diversas dificuldades e desafios provocados pela doença renal e seu tratamento (GRASSELLI et al., 2012).

A adaptação do paciente também variou conforme o ponto de vista sobre o tratamento. Alguns aceitaram com facilidade por notarem as mudanças positivas, principalmente na saúde e bem-estar: "Mudou, porque eu estava me sentindo muito mal e de repente eu comecei a me sentir muito bem, só fez bem na minha vida [...] Eu gostei desde o começo, porque de imediato a gente começa a melhorar e eu amo a hemodiálise" (P10).

Outros passaram por uma adaptação mais conturbada, principalmente durante o primeiro ano, devido às cirurgias para a instalação do cateter e da fístula que incomodam e alteram a aparência física do paciente. Um segundo fator agravante são as diversas restrições impostas pelo tratamento, sobretudo nutricionais e nos hábitos de vida: "Mudou muito porque o que eu fazia eu não posso fazer mais [...] viajar, se alimentar melhor, tomar líquido [...] é só um litro d'água por dia ou menos [...] aí a diferença é muita. A adaptação demorou uns seis meses, quando eu estava com o cateter no pescoço, não podia molhar. Ficar seis meses tomando banho só do peito pra baixo é difícil' (P11).

Buscou-se verificar junto aos pacientes quais medidas podem ser tomadas visando à diminuição da sobrecarga sobre a QV de pacientes renais. Entre as medidas mais citadas estava a criação de uma casa de apoio que recepcionasse 
aqueles que necessitam do tratamento e residem nas cidades circunvizinhas. Os demais fatores citados, com vistas a melhorar as condições relacionadas ao tratamento, foram a facilidade no acesso às informações que poderiam ser mais bem disponibilizadas pelo profissional responsável, bem como o desenvolvimento de pesquisas que possibilitem um menor tempo de diálise e do calibre da agulha utilizada na pulsão venosa, etc. Outros pacientes aguardavam apenas o transplante pois acreditavam que seria a única forma de diminuir os impactos sobre a QV. Esse anseio justifica-se pelo fato do transplante renal melhorar expressivamente a QV da pessoa com IRC, principalmente no aspecto físico (SANTOS \& ROCHA, 2014).

Desta forma, o modo como o paciente encara o tratamento pode definir também sua QV uma vez que diante de uma visão positivista, o paciente busca formas de amenizar as restrições impostas pela hemodiálise, diminuindo os impactos negativos sobre todos os aspectos analisados, principalmente, os emocionais. Logo, o ideal seria que mais informações fossem disponibilizadas aos pacientes, principalmente nos anos iniciais do tratamento, para que estes se familiarizem com sua nova rotina.

Esta pesquisa apresenta como limitação a sua característica transversal, uma vez que a relação de causa e efeito entre as variáveis para a comparação dos dados, antes e após o início do tratamento, não pode ser verificada com confiabilidade. Sendo assim, trabalhos futuros prospectivos podem complementar os dados obtidos com informações referentes às alterações ocorridas durante 0 tratamento, detectando os principais fatores que podem predizer a QV.

\section{CONCLUSÃO}

De forma geral, os pacientes em hemodiálise apresentam elementos que indicam boa qualidade de vida. Entretanto, é importante salientar que a QV é multidimensional e o uso combinado de abordagens quantitativas e qualitativas são importantes para uma avaliação mais precisa desse aspecto. Os resultados indicam que não foi possível predizer a variabilidade na $\mathrm{QV}$, baseado nos determinantes avaliados. Diante de uma visão positivista, o paciente busca formas de amenizar as restrições impostas pela hemodiálise, diminuindo os impactos negativos sobre todos os aspectos analisados, principalmente, os emocionais. Logo, seria de grande importância a disponibilização de informações aos pacientes, especialmente nos anos iniciais do tratamento, para que estes se familiarizem com sua nova rotina e adapte-se mais rapidamente, contribuindo para a melhora da sua QV. Desta forma, é importante que as pessoas em contato com os pacientes em hemodiálise estejam atentos às suas necessidades, sejam físicas e/ou psicológicas. Ações educativas e de apoio ao paciente são necessárias para a promoção da saúde e da QV a fim de minimizar as limitações e problemas decorrentes do tratamento.

\section{AGRADECIMENTOS}

Agradecemos ao enfermeiro Linus Francisco Teixeira e a todos os funcionários da Clínica de Diálise do Norte (CLINORTE) - Porangatu/GO, em especial, à enfermeira Sandra da Silva Moraissem pelo auxílio na obtenção das informações para a realização desta pesquisa. Também agradecemos a todos os pacientes que tão prontamente se disponibilizaram a compartilhar as informações sobre o tratamento. FBT é bolsista do CNPq (302158/2015-4). 


\section{REFERÊNCIAS}

ALVARES, J.; ALMEIDA, A. M.; SZUSTER, D. A. C.; GOMES, I. C.; ANDRADE, E. I. G.; ACURCIO, F. A.; CHERCHIGLIA, M. L. Fatores associados à qualidade de vida de pacientes em terapia renal substitutiva no Brasil. Ciência \& Saúde Coletiva, v. 18, n. $7, \quad$ p. 1903-1910, 2013. Disponível em: $<$ http://www.scielo.br/scielo.php?pid=S1413-

$81232013000700005 \&$ script=sci abstract\&tlng=pt $>$. doi: http://dx.doi.org/10.1590/S1413-81232013000700005.

BARBOSA, G. S.; VALADARES, G. Hemodiálise: estilo de vida e adaptação do paciente. Acta Paulista de Enfermagem, v. 22, n. 5, p. 24-27, 2009. Disponível em: $<$ http://www.scielo.br/scielo.php?script=sci_arttext\&pid=S0103-

21002009000800014>. doi: http://dx.doi.org/10.1590/S0103-21002009000800014.

BASTOS, M. G.; BREGMAN, R.; Kirsztajn, G. M. Doença renal crônica: frequente e grave, mas também prevenível e tratável. Revista da Associação Médica Brasileira, v. 56, n. 2, p. 248-53, 2010. Disponível em: $<$ http://www.scielo.br/scielo.php?script=sci_arttext\&pid=S010442302010000200028>. doi: http://dx.doi.org/10.1590/S0104-42302010000200028.

BERLIM M. T.; PAVANELLO, D. P.; CALDIERARO, M. A. K. FLECK MP. Reliability and validity of the WHOQOL BREF in a sample of Brazilian outpatients with major depression. Quality of Life Research, v. 14, n. 2, p. 561-564, 2005. Disponível em: $<$ https://www.ncbi.nlm.nih.gov/pubmed/15892446>.

BRASIL. Ministério da Saúde. Política Nacional de Atenção ao Portador de Doença Renal. Portaria GM/SM n. 1168, de 15 de junho de 2004. [acesso em: 22 fev. 2013].

Disponível em: <http://dtr2001.saude.gov.br/sas/PORTARIAS/Port2004/GM/GM1168.htm>.

CASTRO, E. K.; GROSS, C. Q. Percepção sobre a doença renal crônica de pacientes em hemodiálise: revisão sistemática. Salud \& Sociedad, v. 4, n. 1, p. 7089 , 2013. Disponível $<$ https://dialnet.unirioja.es/servlet/articulo?codigo $=4323213>$.

DRAIBE, S. A. Panorama da Doença Renal Crônica no Brasil e no Mundo. São Luís: Universidade Federal do Maranhão. UNASUS/UFMA, 2014. Disponível em: <file://C:/Users/luond/Downloads/modulo_3_unidade_1.pdf >.

FERMI, M. R. V. Manual de diálise para enfermagem. Rio de Janeiro: MEDSI, 2003.

GARCIA-GARCIA, G.; HAREN, P.; CHAPMAN, J. The Global Role of Kidney Transplantation. Kidney Blood Pressure Research, v. 35, p. 299-304, 2012. Disponível em: http://www.karger.com/Article/Abstract/337044. doi: 10.1159/000337044. 
GRASSELLI, C. S. M.; CHAVES, E. C. L.; SIMÃO, T. P.; BOTELHO, P. B.; SILVA, R. R. Avaliação da qualidade de vida dos pacientes submetidos à hemodiálise. Revista da Sociedade Brasileira de Clinica Médica, v. 10, n. 6, p. 503-507, 2012. Disponível em: < http://files.bvs.br/upload/S/1679-1010/2012/v10n6/a3185.pdf >.

LI, P. K. T.; BURDMANN, E. A.; MEHTA, R. L. Acute Kidney Injury: a global alert. Jornal Brasileiro de Nefrologia. v. 35, n. 1, p. 1-5, 2013. Disponível em: <http://jbn.org.br/details/1522/en-US/acute-kidney-injury--a-global-alert>. $\quad$ doi: http://dx.doi.org/10.5935/01012800.20130001 .

LIMA, A. F. C.; GUALDA, D. M. R. História oral de vida: buscando o significado da hemodiálise para o paciente renal crônico. Revista da Escola de Enfermagem da USP, v. $35, \quad$ n. 3 , p. 235-241, 2001. Disponível em: $<$ http://www.scielo.br/scielo.php?pid=S0080-

$62342001000300006 \&$ script=sci_abstract\&tIng=pt $>$.

doi:

http://dx.doi.org/10.1590/S0080-62342001000300006.

LIRA, C. L. O. B.; AVELAR, T. C.; BUENO, J. M. M. H. COPING E QUALIDADE DE VIDA DE PACIENTES EM HEMODIÁLISE. Estudos Interdisciplinares em Psicologia, v. $6, \quad$ n. $1, \quad$ p. $82-99, \quad 2015$. Disponível em: <http://www.uel.br/revistas/uel/index.php/eip/article/view/21464>. doi: http://dx.doi.org/10.5433/2236-6407.2015v6n1p82.

LOPES, J. M. FUKUSHIMA, R. L. M.; INOUYE, K.; PAVARINI, S. C. I; ORLANDI, F. $S$. Qualidade de vida relacionada à saúde de pacientes renais crônicos em diálise. Acta Paulista de Enfermagem, v. 2, n. 3, p. 230-236, 2014. Disponível em: <http://www.scielo.br/pdf/ape/v27n3/1982-0194-ape-027-003-0230.pdf>. doi: http://dx.doi.org/10.1590/1982-0194201400039.

MACHADO, G. R. G.; PINHATI, F. R. Tratamento de diálise em pacientes com insuficiência renal crônica. Cadernos UniFOA, v. 26, n. 1, 2014. Disponível em: $<$ www.unifoa.edu.br/cadernos/ojs $>$.

MADEIRO, A. C.; MACHADO, P. D. L. C.; BONFIM, I. M.; BRAQUEAIS, A. R.; LIMA, F. E. T. Adesão de portadores de insuficiência renal crônica ao tratamento de hemodiálise. Acta Paulista de Enfermagem, v. 23, n. 4, p. 546-551, 2010. Disponivel em: <http://www.scielo.br/scielo.php?pid=S0103$21002010000400016 \&$ script=sci_abstract\&tlng $=\mathrm{pt} />$. doi: http://dx.doi.org/10.1590/S0103-21002010000400016.

MARTELLI, A. Sistema Renal e sua Influência no Controle em Longo Prazo da Pressão Arterial. UNOPAR Cientifíca. Ciências Biológicas e da Saúde, v. 15, n. p. 75-80, 2013. Disponível em: $\square$ http://pgsskroton.com.br/seer/index.php/JHealthSci/article/view/797/762 $\square$. doi: http://dx.doi.org/10.17921/2447-8938.2013v15n1p\%25p. 
MARTINS, M. R. I.; CESARINO, C. B. Qualidade de vida de pessoas com insuficiência renal crônica em tratamento hemodialítico. Revista Latino-Americana de Enfermagem, v. 13, n. 5, p. 670-676, 2005. Disponível em: $<$ http://bvsms.saude.gov.br/bvs/is_digital/is_0106/pdfs/IS26(1)017.pdf>.

MEDEIROS, A. J. S.; MEDEIROS E. M. D. A assistência de enfermagem prestada no tratamento hemodialítico promovido junto ao portador de insuficiência renal crônica - Uma revisão de literatura. Revista Brasileira de Educação e Saúde, v. 3, n. $2, \quad$ p. $13-17,2013 . \quad$ Disponível em: <http://www.gvaa.com.br/revista/index.php/REBES/article/view/2129 >.

Pedroso, B.; PILATTI, L. A.; GUTIERREZ, G. L.; PICININ, C. T. Cálculo dos escores e estatística descritiva do WHOQOL-bref através do Microsoft Excel. Revista Brasileira de Qualidade de Vida. v. 2, n. 1, p. 31-36, 2010. Disponível em: <https://periodicos.utfpr.edu.br/rbqv/article/view/687>. doi. 10.3895/S217508582010000100004.

RIBEIRO, I. P.; PINHEIRO, A. L. S.; SOARES, A. L. A.; SANTOS, N. F. M. Perfil Epidemiológico dos portadores de insuficiência renal crônica submetidos à terapia hemodialítica. Enfermagem em Foco. v. 5, p. 65-69, 2014.

ROCHA, N. S.; FLECK, M. P. A. Validity of the Brazilian version of WHOQOL-BREF in depressed patients using Rasch modelling. Revista de Saúde Pública, v. 43, n.1, 2009. Disponível em: <http://www.scielo.br/pdf/rsp/v43n1/7240.pdf>.

SANTOS, P. R. Relação do sexo e da idade com nível de qualidade de vida em renais crônicos hemodialisados. Revista da Associação Médica Brasileira, v. 52, n. 5, p. 356-359, 2006. Disponível em: < http://www.scielo.br/scielo.php?pid=S010442302006000500026\&script=sci_abstract\&tlng=pt>.

doi: http://dx.doi.org/10.1590/S0104-42302006000500026.

SANTOS, P. R.; ROCHA, D. L. B. Qualidade de vida pós-transplante renal: revisão integrativa. Enfermería Nefrológica, v. 17, n. 1, p. 51-58, 2014. Disponível em: $<$ http://scielo.isciii.es/scielo.php?pid=S2254-

28842014001100009\&script=sci_abstract\&tlng=pt>.

SESSO, R. C.; LOPES, A. A.; THOMÉ, F. S.; LUGON, J. R.; MARTINS, C. T. Inquérito Brasileiro de Diálise Crônica 2014. Jornal Brasileiro de Nefrologia, v. 38, n. 1, p. 54-61, 2016. Disponível em: <http://www.scielo.br/pdf/jbn/v38n1/0101-2800jbn-38-01-0054.pdf>. doi: 10.5935/0101-2800.20160009.

SILVA, A. S.; SILVEIRA, R. S.; FERNANDES, G. F. M.; LUNARDI, V. L.; BACKES, V. M. S. Percepções e mudanças na qualidade de vida de pacientes submetidos à hemodiálise. Revista Brasileira de Enfermagem, v. 64, n. 5, p. 839-844, 2011. Disponível em: <http://www.scielo.br/scielo.php?script=sci_arttext\&pid=S003471672011000500006>. doi: http://dx.doi.org/10.1590/S0034-71672011000500006. 
SINGER M. A.; HOPMAN, W. M.; MACKENZIE, T. A. Physical functioning and mental health in patients with chronic medical conditions. Quality of Life Research, v. 8, n. 8, p.687-91, 1999. Disponível em: <https://www.ncbi.nlm.nih.gov/pubmed/10855342>.

SOCIEDADE BRASILEIRA DE NEFROLOGIA (Brasil). Tratamento. São Paulo, 2016. [acesso em 13 set. 2016]. Disponível em: <http://www.sbn.org.br/>.

TELLES, C. T.; DOBNER, T.; POMATTI, G.; FORTES, V. F.; BROCK, F.; BETTINELLI, L. A. Perfil sociodemográfico, clínico e laboratorial de pacientes submetidos à hemodiálise. Revista Rene. v. 15, n. 3, p. 420-426, 2014. Disponível em: <file:///C:/Users/luond/Downloads/1564-11777-1-PB.pdf>. doi: 10.15253/21756783.2014000300006.

THE WHOQOL GROUP. Development of the World Health Organization WHOQOLBREF quality of life assessment. Psychological Medicine, v. 28, n. 3, p. 551-558, 1998. Disponível em: https://www.ncbi.nlm.nih.gov/pubmed/9626712.

VALLE, L. D. S.; SOUZA, V. F. D.; RIBEIRO, A. M. Estresse e ansiedade em pacientes renais crônicos submetidos à hemodiálise. Estudos em Psicologia (Campinas), v. 30, n. 1, p. 131-38, 2013. Disponível: <http://www.scielo.br/scielo.php?pid=S0103166X2013000100014\&script=sci_abstract>. doi: http://dx.doi.org/10.1590/S0103$166 \times 2013000100014$.

VIEIRA JÚNIOR, J. M.; SUASSUNA, J. H. R. O acometimento renal na hipertensão arterial e diabetes mellitus tipo 2: como identificar e prevenir. A visão do nefrologista. Revista Hospital Universitário Pedro Ernesto, v. 12, p. 53-60, 2013. Disponível em: <http://revista.hupe.uerj.br/detalhe_artigo.asp?id=407>. 